\title{
How Prof. Zeidler Supported Our Research on Exact Solution of Quantum Field Theory Toy Models
}

\author{
Harald Grosse $^{1}$ (D) $\cdot$ Raimar Wulkenhaar ${ }^{2}$
}

Received: 27 December 2017 / Accepted: 6 April 2018 / Published online: 6 September 2018

(C) The Author(s) 2018

\begin{abstract}
Over many years, we developed the construction of the $\phi^{4}$-model on four-dimensional Moyal space. The solution of the related matrix model $\mathcal{Z}[E, J]=\int d \Phi \exp \left(\operatorname{tr}\left(J \Phi-E \Phi^{2}-\right.\right.$ $\left.\frac{\lambda}{4} \Phi^{4}\right)$ ) is given in terms of the solution of a non-linear equation for the 2-point function and the eigenvalues of $E$. The resulting Schwinger functions in position space are symmetric and invariant under the full Euclidean group. Locality is fulfilled. The Schwinger 2-point function is reflection positive in special cases.
\end{abstract}

Keywords 4D noncommutative quantum field theory · Renormalisation · Non-perturbative solution · Integral equation

PACS 2010 11.10.Gh $\cdot 11.10 . \mathrm{Lm} \cdot 02.30 . \mathrm{Rz}$

\section{Introduction}

Professor Zeidler supported and influenced our common work over many years: During the one-semester stay in 2000/2001 of one of us (HG), we enjoyed the friendly hospitality at MPI Leipzig. On the other hand, from 2002 to 2005, RW was post-doc at the MPI. During this time, we achieved the perturbative renormalisation proof of the noncommutative $\phi_{4}^{4}$ model. Prof. Zeidler was constantly interested in the progress of this work, financed our mutual visits in Leipzig and Vienna and, most importantly, introduced Vincent Rivasseau to

Dedicated to the memory of Prof. Eberhard Zeidler (1940-2016).

\footnotetext{
Harald Grosse

harald.grosse@univie.ac.at

Raimar Wulkenhaar

raimar@math.uni-muenster.de
}

1 Fakultät für Physik, Universität Wien, Boltzmanngasse 5, A-1090, Wien, Austria

2 Mathematisches Institut der Westfälischen Wilhelms-Universität, Einsteinstraße 62,

D-48149, Münster, Germany 
RW in December 2003. This changed life of all three of us. Vincent jumped to the subject and pushed it enormously, together with his group. They extended the vanishing of the beta-function to all orders in pertubation theory and led us to develop the non-perturbative solution after 2009. On the first version of these equations, we had an intense exchange with Prof. Zeidler in 2011. These discussions inspired our reduction of the equations to a fixed point problem in 2012. In a contribution to a special volume dedicated to Prof. Zeidler's 75 th birthday, we were able to prove existence of a solution. We remember the long phone conversation with Prof. Zeidler about this fixed point problem.

In this contribution, we summarise the main developments in our programme since 2002 , referring to [8-16]. Originally intended as renormalisation of the noncommutative $\phi_{4}^{4}$-model, our work turned into a programme to exactly solve quantum field theory toy models:

1. In December 1999, Minwalla, van Raamsdonk, and Seiberg pointed out [19] that Feynman graphs for scalar fields on noncommutative $\mathbb{R}^{4}$ show a novel type of singularity (termed UV/IR-mixing) which prevented renormalisation. This came as big surprise, and for several years, no solution was found. Eventually, combining the WilsonPolchinski programme for noncommutative $\phi^{4}$-theory with the harmonic oscillator base of the Moyal plane (which avoids the phase factors appearing in momentum space), we solved in a series of papers [9-11] the renormalisation problem. Thereby, we achieved the remarkable balance of proving renormalisability of the $\phi^{4}$-model to all orders and reconfirming the UV/IR-duality of [19]. The main steps are given in Section 2.

2. Soon after the renormalisation proof, we showed that the running coupling constant has bounded flow to one-loop order (see [8], reviewed in Section 3). This result led to a close collaboration with Vincent Rivasseau and his group. He emphasised that it should be possible to construct this model non-perturbatively! They established the foundation by proving that at a special self-duality point, the $\beta$-function vanishes to all orders [5]. We understood that their method goes beyond the $\beta$-function and used it to derive a closed non-perturbative equation for the 2-point function [12] (which we intensely discussed with Prof. Zeidler).

3. In [14], we gave a rigorous derivation of these equations. As reviewed in Section 4, Ward identities for the $U(\infty)$ group action lead to an exact solution of the quartic matrix model in terms of the solution of a non-linear equation. As by-product, we find that any renormalisable quartic matrix model has vanishing $\beta$-function.

4. Self-dual $\phi_{4}^{4}$-theory on Moyal space $[10,11]$ is of that type. We give a summary of the proof in Section 4.3. The non-perturbative solution leads, for extreme noncommutativity $\theta \rightarrow \infty$, and after careful discussion of thermodynamic and continuum limit, to a non-linear fixed point equation [14], for which a non-perturbative and non-trivial solution exists for $\lambda<0$ [16]. The key step is the observation that a certain difference function satisfies a linear singular integral equation of Carleman type.

5. Following [13], we identify in Section 5 a limit to Schwinger functions for a scalar field on $\mathbb{R}^{4}$. Surprisingly for a highly noncommutative model, these Schwinger functions show full Euclidean symmetry. Otherwise, they have unusual properties such as absent momentum transfer in interaction processes. This seems to suggest triviality, but the numerical investigation [15] of the 2-point function shows scattering remnants from a noncommutative geometrical substructure. Most surprisingly, the Schwinger 2-point function seems to be reflection positive in one of its phases. 


\section{Renormalisation of Noncommutative $\phi^{4}$-theory to All Orders}

The renormalised $\phi^{4}$-model corresponds to the classical action

$$
S=\int d^{4} x\left(\frac{1}{2} \partial_{\mu} \phi \star \partial^{\mu} \phi+\frac{\Omega^{2}}{2}\left(\tilde{x}_{\mu} \phi\right) \star\left(\tilde{x}^{\mu} \phi\right)+\frac{\mu^{2}}{2} \phi \star \phi+\frac{\lambda}{4} \phi \star \phi \star \phi \star \phi\right)(x),
$$

with $\tilde{x}_{\mu}:=2\left(\Theta^{-1}\right)_{\mu \nu} x^{\nu}$ and the star product is defined by $[6,7]$ :

$$
(a \star b)(x)=\int \frac{d^{D} y d^{D} k}{(2 \pi)^{D}} a\left(x+\frac{1}{2} \Theta \cdot k\right) b(x+y) \mathrm{e}^{\mathrm{i} k y} .
$$

The appearance of the harmonic oscillator term $\frac{\Omega^{2}}{2}\left(\tilde{x}_{\mu} \phi\right) \star\left(\tilde{x}^{\mu} \phi\right)$ in the action (1) is a result of the renormalisation proof, as sketched below. It also permits a transformation

$$
S\left[\phi ; \mu_{0}, \lambda, \Omega\right] \mapsto \Omega^{2} S\left[\phi ; \frac{\mu_{0}}{\Omega}, \frac{\lambda}{\Omega^{2}}, \frac{1}{\Omega}\right]
$$

under Langmann-Szabo duality [17].

Relativistic quantum field theories on noncommutative Minkowski space are much more difficult [1]. Here the UV/IR-mixing problem occurs in different types of graphs [2].

\subsection{The $\phi^{4}$-action in the Matrix Base}

We assume for simplicity that $\theta_{12}=-\theta_{21}=\theta_{34}=-\theta_{43}$ are the only non-vanishing components. A key step is expansion of the fields in the harmonic oscillator base [7] which in two dimensions reads

$$
f_{m n}(z)=2(-1)^{m} \sqrt{\frac{m !}{n !}}\left(\sqrt{\frac{2}{\theta}} z\right)^{n-m} L_{m}^{n-m}\left(\frac{2|z|^{2}}{\theta}\right) e^{-\frac{|z|^{2}}{\theta}}
$$

with $z \in \mathbb{C} \equiv \mathbb{R}^{2}$. Collecting them to $f_{\underline{m} \underline{n}}\left(x_{1}, \ldots, x_{4}\right)=f_{m_{1} n_{1}}\left(x_{1}+\mathrm{i} x_{2}\right) f_{m_{2} n_{2}}\left(x_{3}+\mathrm{i} x_{4}\right)$ where $\underline{m}={ }_{m^{2}}{ }^{2}$, these functions satisfy $\left(f_{\underline{m} \underline{n}} \star f_{\underline{k l}}\right)(x)=\delta_{\underline{n k}} f_{\underline{m l}}(x)$ and $\int_{\mathbb{R}^{4}} d x f_{\underline{m} \underline{n}}(x)=$ $(2 \pi \theta)^{2} \delta_{\underline{m} \underline{n}}$. Working out the action of Laplace operator and multiplication by $x$ on $\underline{f}_{\underline{m} \underline{n}}$ and expanding $\phi(x)=\sum_{\underline{m}, \underline{n}} \Phi_{\underline{m} \underline{n}} f_{\underline{m} \underline{n}}(x)$, the action (1) takes the form

$$
\begin{aligned}
& S[\phi] \equiv S[\Phi]=(2 \pi \theta)^{2} \sum_{\underline{m}, \underline{n}, \underline{k}, \underline{l} \in \mathbb{N}^{2}}\left(\frac{1}{2} \Phi_{\underline{m} \underline{n}} \Delta_{\underline{m} \underline{n} ; \underline{k} \underline{l}} \Phi_{\underline{k} \underline{l}}+\frac{\lambda}{4} \Phi_{\underline{m} \underline{n}} \Phi_{\underline{n k}} \Phi_{\underline{k} \underline{l}} \Phi_{\underline{l m}}\right),
\end{aligned}
$$

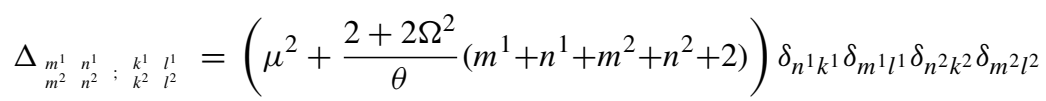

$$
\begin{aligned}
& -\frac{2-2 \Omega^{2}}{\theta}\left(\sqrt{k^{1} l^{1}} \delta_{n^{1}+1, k^{1}} \delta_{m^{1}+1, l^{1}}+\sqrt{m^{1} n^{1}} \delta_{n^{1}-1, k^{1}} \delta_{m^{1}-1, l^{1}}\right) \delta_{n^{2} k^{2}} \delta_{m^{2} l^{2}} \\
& -\frac{2-2 \Omega^{2}}{\theta}\left(\sqrt{k^{2} l^{2}} \delta_{n^{2}+1, k^{2}} \delta_{m^{2}+1, l^{2}}+\sqrt{m^{2} n^{2}} \delta_{n^{2}-1, k^{2}} \delta_{m^{2}-1, l^{2}}\right) \delta_{n^{1} k^{1}} \delta_{m^{1} l^{1}} .
\end{aligned}
$$

The quantum field theory is constructed as a perturbative expansion about the free theory, which is solved by the propagator $G_{\underline{m} \underline{\underline{n}} ; \underline{\underline{k}},}$, the inverse of $\Delta_{\underline{m} \underline{\underline{n}} ; \underline{\underline{l}} .}$. After diagonalisation 
of $\Delta_{m n ; k l}$ (which leads to orthogonal Meixner polynomials) and the use of identities for hypergeometric functions one arrives at

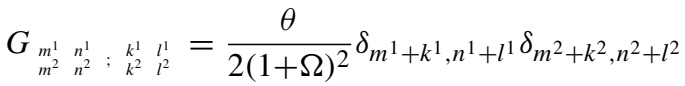

$$
\begin{aligned}
& \times \sum_{v^{1}=\frac{\left|m^{1}-l^{1}\right|}{2}}^{\frac{m^{1}+l^{1}}{2}} \sum_{v^{2}=\frac{\left|m^{2}-l^{2}\right|}{2}}^{\frac{m^{2}+l^{2}}{2}} B\left(1+\frac{\mu^{2} \theta}{8 \Omega}+\frac{1}{2}\left(m^{1}+k^{1}+m^{2}+k^{2}\right)-v^{1}-v^{2}, 1+2 v^{1}+2 v^{2}\right) \\
& \times_{2} F_{1}\left(\begin{array}{l|l}
1+2 v^{1}+2 v^{2}, \frac{\mu^{2} \theta}{8 \Omega}-\frac{1}{2}\left(m^{1}+k^{1}+m^{2}+k^{2}\right)+v^{1}+v^{2} & \frac{(1-\Omega)^{2}}{(1+\Omega)^{2}}
\end{array}\right) \\
& \times\left(\frac{1-\Omega}{1+\Omega}\right)^{2 v^{1}+2 v^{2}} \\
& \times \prod_{i=1}^{2} \sqrt{\left(\begin{array}{c}
n^{i} \\
v^{i}+\frac{n^{i}-k^{i}}{2}
\end{array}\right)\left(\begin{array}{c}
k^{i} \\
v^{i}+\frac{k^{i}-n^{i}}{2}
\end{array}\right)\left(\begin{array}{c}
m^{i} \\
v^{i}+\frac{m^{i}-l^{i}}{2}
\end{array}\right)\left(\begin{array}{c}
l^{i} \\
v^{i}+\frac{l^{i}-m^{i}}{2}
\end{array}\right)} .
\end{aligned}
$$

It is important that the sums in (5) are finite.

\subsection{Renormalisation Group Approach to Dynamical Matrix Models}

The (Euclidean) quantum field theory is defined by the partition function

$$
\mathcal{Z}[J]=\int \mathcal{D}[\phi] \exp \left(-S[\Phi]-(2 \pi \theta)^{2} \sum_{\underline{m}, \underline{n}} \Phi_{\underline{m} \underline{\underline{n}}} J_{\underline{n} \underline{m}}\right) .
$$

The idea inspired by Polchinski's renormalisation proof of commutative $\phi^{4}$-theory is to change the weights of the matrix indices in the kinetic part of $S[\Phi]$ as a smooth function of an energy scale $\Lambda$ and to compensate this by a careful adaptation of the effective action $L[\Phi, \Lambda]$ such that $\mathcal{Z}[J]$ becomes independent of the scale $\Lambda$. If the modification of the weights of a matrix index $m \in \mathbb{N}$ is described by a function $K\left(\frac{m}{\theta \Lambda^{2}}\right)$, then the required $\Lambda$-dependence of the effective action is given by the matrix Polchinski equation

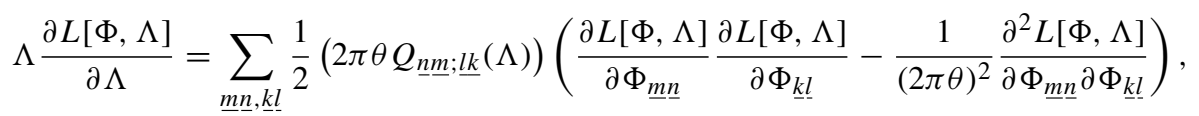

where

$$
2 \pi \theta Q_{\underline{n} \underline{m} ; \underline{l k}}(\Lambda):=\Lambda \frac{\partial}{\partial \Lambda}\left(\prod_{i \in m^{1}, m^{2}, \ldots, l^{1}, l^{2}} K\left(\frac{i}{\theta \Lambda^{2}}\right) G_{\underline{n} \underline{m} ; \underline{l k}}(\Lambda)\right) .
$$

In this section, we look for a perturbative solution of the matrix Polchinski equation (6). In terms of the expansion coefficients

$$
L[\Phi, \Lambda]=\sum_{V=1}^{\infty} \lambda^{V} \sum_{N=2}^{2 V+2} \frac{(2 \pi \theta)^{\frac{N}{2}-2}}{N !} \sum_{\underline{m}_{i}, \underline{n}_{i} \in \mathbb{N}^{2}} A_{\underline{m}_{1} \underline{n}_{1} ; \ldots ; \underline{m}_{N} \underline{n}_{N}}^{(V)}[\Lambda] \Phi_{\underline{m}_{1} \underline{n}_{1}} \cdots \phi_{\underline{m}_{N} \underline{n}_{N}}
$$


of the effective action, the matrix Polchinski equation (6) is represented by ribbon graphs (we suppress $\underline{n} \mapsto n$ in ribbon graphs):

$$
\begin{aligned}
& \Lambda \frac{\partial}{\partial \Lambda} \vdots \overbrace{\overbrace{m_{2}} i^{n_{2}}}^{n_{1} m_{N}}
\end{aligned}
$$

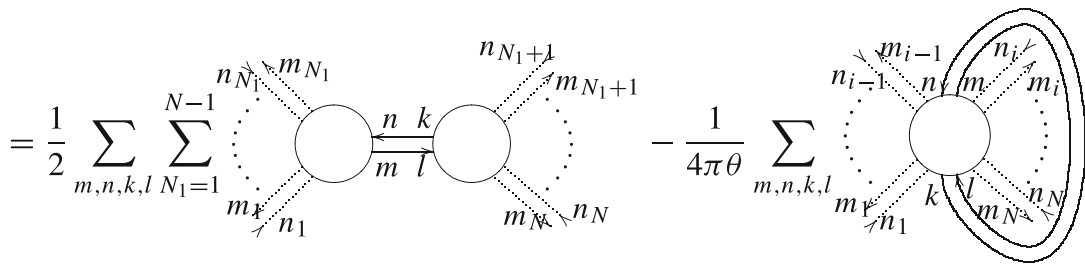

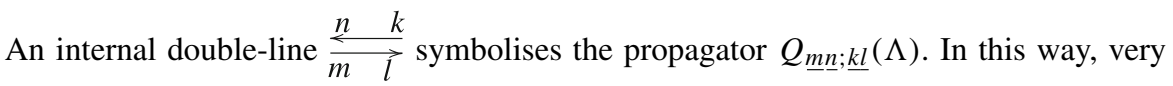
complicated ribbon graphs can be produced which cannot be drawn any more in a plane. A ribbon graph represents a simplicial complex for a Riemann surface and thus defines the topology of the Riemann surface on which it can be drawn. The Riemann surface is characterised by its genus $g$ computable via the Euler characteristic of the graph, $g=$ $1-\frac{1}{2}(L-I+V)$, and the number $B$ of holes. Here, $L$ is the number of single-line loops if we close the external lines of the graph, $I$ is the number of double-line propagators and $V$ the number of vertices. The number $B$ of holes coincides with the number of single-line cycles which carry external legs. Accordingly, we also label the expansion coefficients in (8) by the topology, $A_{\underline{m}_{1}, \underline{n}_{1} ; \ldots ; \underline{m}_{N} \underline{n}_{N}}^{(V, B, g)}$.

We have proven in [9] a power-counting estimation for these coefficients which relates the $\Lambda$-scaling of a ribbon graph to the topology of the graph and to two asymptotic scaling dimensions of the differentiated cut-off propagator $Q_{\underline{m} \underline{n} ; \underline{k} \underline{\underline{k}}}(\Lambda)$. As a result, if these scaling dimensions coincide with the classical momentum space dimensions, then all nonplanar graphs are suppressed by the renormalisation flow. This is a necessary requirement for the renormalisability of a model. On the other hand, as the expansion coefficients $A_{\underline{m}_{1} \underline{n}_{1} ; \ldots ; \underline{m}_{N} \underline{n}_{N}}^{(V)}[\Lambda]$ carry an infinite number of matrix indices, the general power-counting estimation proven in [9] leaves, a priory, an infinite number of divergent planar graphs. These planar graphs require a separate analysis.

\subsection{Power-Counting Behaviour of the Noncommutative $\phi^{4}$-model}

The key is the integration procedure of the Polchinski equation (9), which involves the entire magic of renormalisation. We consider the example of the planar one-particle irreducible four-point function with two vertices, $A_{\underline{m}_{1} \underline{n}_{1} ; \ldots ; \underline{m}_{4} \underline{n}_{4}}^{(2,1,0) 1 \mathrm{PI}}$. The Polchinski equation (9) provides the $\Lambda$-derivative of that function:

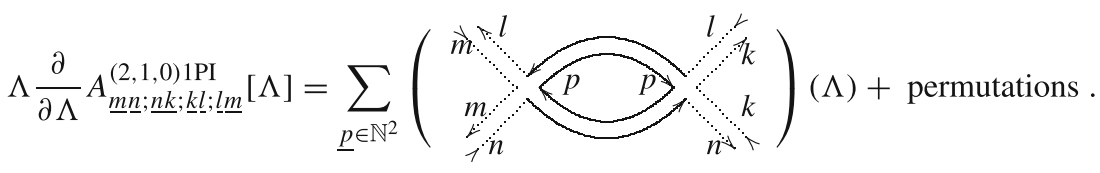


Performing the $\Lambda$-integration of (10) from some initial scale $\Lambda_{0}$ (sent to $\infty$ at the end) down to $\Lambda$, we obtain $A_{\underline{m} \underline{n} ; \underline{n} k ; \underline{k}, \underline{l} ; \underline{\underline{m}}}^{(2,1,0) 1 \mathrm{PI}}[\Lambda] \sim \log \frac{\Lambda_{0}}{\Lambda}$, which diverges for $\Lambda_{0} \rightarrow \infty$. Renormalisation can be understood as the change of the boundary condition for the integration.

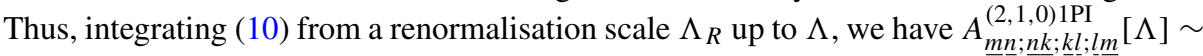
$\log \frac{\Lambda}{\Lambda_{R}}$, and there would be no problem for $\Lambda_{0} \rightarrow \infty$. However, since there is an infinite number of matrix indices and there is no symmetry which could relate the amplitudes, that

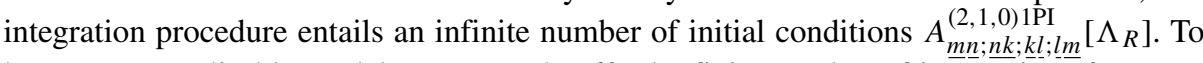
have a renormalisable model, we can only afford a finite number of integrations from $\Lambda_{R}$ up to $\Lambda$. Thus, the correct choice is

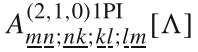

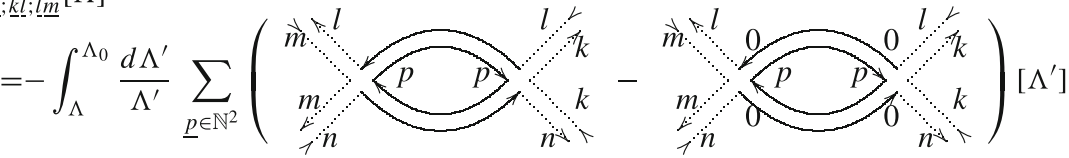

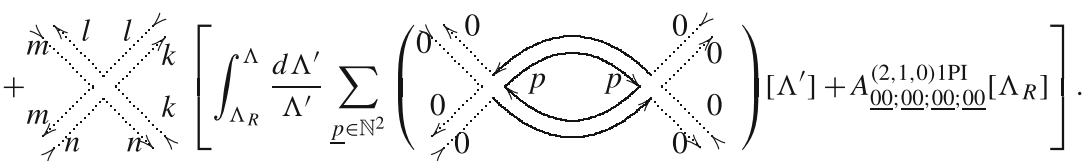

The second graph in the first line on the right-hand side and the graph in brackets in the last line are identical, because only the indices on the propagators determine the value of the graph. Moreover, the vertex in the last line in front of the bracket equals 1 . Thus, differentiating (11) with respect to $\Lambda$ we obtain indeed (10). As a further check one can consider (11) for $\underline{m}=\underline{n}=\underline{k}=\underline{l}={ }_{0}^{0}$. Finally, the independence of $A_{\underline{m} \underline{n} ; \underline{n} \underline{k} ; \underline{k} \underline{k} ; \underline{l} \underline{m} \underline{m}}^{(2,1,0) 1 \mathrm{I}}\left[\Lambda_{0}\right]$ on the indices $\underline{m}, \underline{n}, \underline{k}, \underline{l}$ is built-in. This property is, for $\Lambda_{0} \rightarrow \infty$, dynamically generated by the model.

There is a similar $\Lambda_{0}-\Lambda_{R}$-mixed integration procedure for the planar 1PI two-point

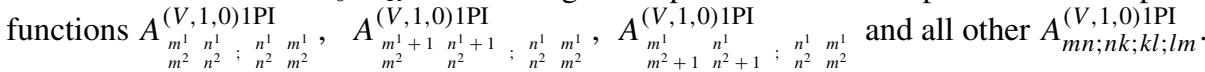
These involve in total four different sub-integrations from $\Lambda_{R}$ up to $\Lambda$. We refer to [10] for details. All other graphs are integrated from $\Lambda_{0}$ down to $\Lambda$, e.g.,

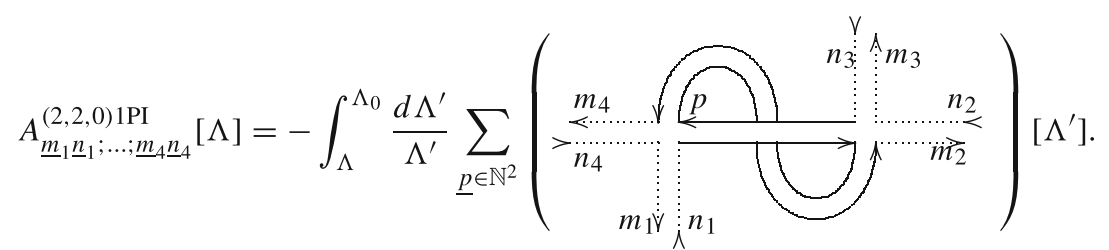

Theorem 1 The previous integration procedure yields

$$
\begin{aligned}
& \mid A_{\underline{m}_{1} \underline{\underline{n}}_{1} ; \ldots ; \underline{m}_{N} \underline{\underline{n}}_{N}[\Lambda] \mid}^{(V, B, g)} P^{4 V-N}\left[\frac{\max \left(\left|\underline{m}_{1}\right|,\left|\underline{n}_{1}\right|, \ldots\left|\underline{n}_{N}\right|\right)}{\theta \Lambda^{2}}\right] P^{2 V-\frac{N}{2}}\left[\log \frac{\Lambda}{\Lambda_{R}}\right],
\end{aligned}
$$

where $P^{q}[X]$ stands for a polynomial of degree $q$ in $X$ and $\left|\begin{array}{l}m^{1} \\ m^{2}\end{array}\right|=m^{1}+m^{2}$. 
Idea of the proof. For the choice $K(x)=1$ for $0 \leq x \leq 1$ and $K(x)=0$ for $x \geq 2$ of the cut-off function in (7) one has

$$
\left|Q_{\underline{m} \underline{n} ; \underline{k} \underline{l}}(\Lambda)\right|<\frac{C_{0}}{\Omega \theta \Lambda^{2}} \delta_{\underline{m}+\underline{k}, \underline{n}+\underline{l}} .
$$

Thus, the propagator and the volume of a loop summation have the same power-counting dimensions as a commutative $\phi^{4}$-model in momentum space, giving the total powercounting degree $4-N$ for an $N$-point function.

This is (more or less) correct for planar graphs. The scaling behavior of non-planar graphs is considerably improved by the quasi-locality of the propagator:

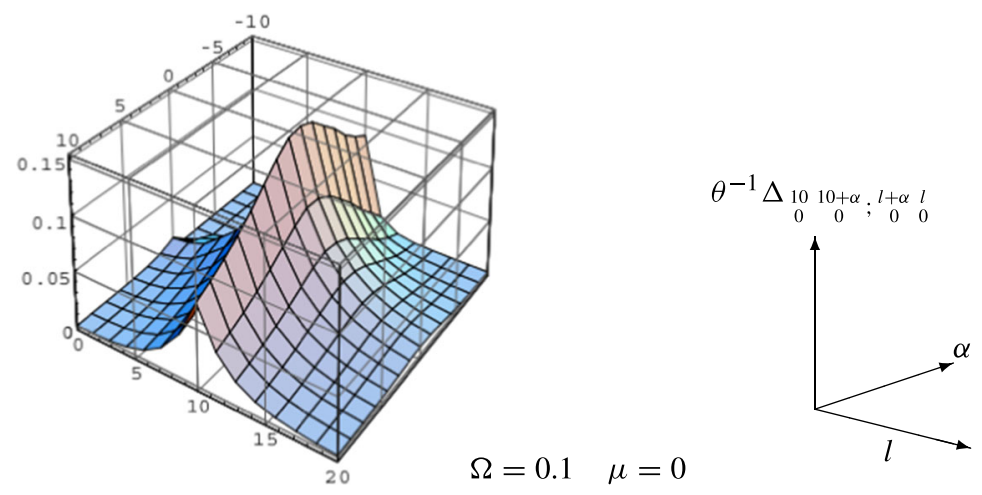

As a consequence, for given index $\underline{m}$ of the propagator $Q_{\underline{m} \underline{n} ; \underline{k} \underline{\underline{l}}}(\Lambda)=\frac{\stackrel{n}{m} \quad k}{\mathrm{l}}$, the contribution to a graph is strongly suppressed unless the other index $\underline{l}$ on the trajectory through $\underline{m}$ is close to $\underline{m}$. Thus, the sum over $\underline{l}$ for given $\underline{m}$ converges and does not alter (apart from a factor $\Omega^{-1}$ ) the power-counting behaviour of (14):

$$
\sum_{\underline{l} \in \mathbb{N}^{2}}\left(\max _{\underline{n}, \underline{k}}\left|Q_{\underline{m} \underline{n} ; \underline{k} \underline{l}}(\Lambda)\right|\right)<\frac{C_{1}}{\theta \Omega^{2} \Lambda^{2}}
$$

In a non-planar graph like the one in (12), the index $\underline{n}_{3}$-fixed as an external indexlocalises the summation index $\underline{p} \approx \underline{n}_{3}$. Thus, we save one volume factor $\theta^{2} \Lambda^{4}$ compared with a true loop summation as in (2.3). In general, each hole in the Riemann surface saves one volume factor, and each handle even saves two.

A more careful analysis of (5) shows that also planar graphs get suppressed with $\left|Q_{\begin{array}{ccc}m^{1} n^{1} \\ m^{2}\end{array} n^{2}: \begin{array}{c}k^{1} \\ k^{2}\end{array} l^{l^{2}}}(\Lambda)\right|<\frac{C_{2}}{\Omega \theta \Lambda^{2}} \prod_{i=1}^{2}\left(\frac{\max \left(m^{i}, l^{i}\right)+1}{\theta \Lambda^{2}}\right)^{\frac{\left|m^{i}-l^{i}\right|}{2}}$, for $m^{i} \leq n^{i}$, if the index along a

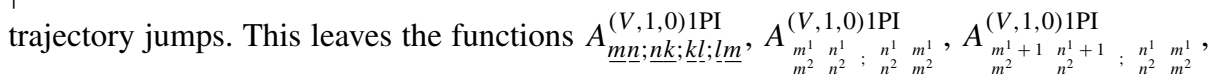
and $A_{\begin{array}{l}m^{1} \\ m^{2}+1\end{array} n^{1}+1}^{(V, 1,0) 1 P I} ; \begin{array}{lll}n^{1} & m^{1} \\ n^{2} & m^{2}\end{array}$ as the only relevant or marginal ones. In these functions one has to use a discrete version of the Taylor expansion such as

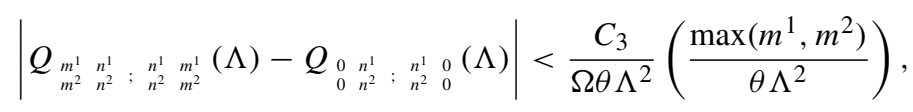


which can be traced back to the Meixner polynomials. The discrete Taylor subtractions are used in the integration from $\Lambda_{0}$ down to $\Lambda$ in prescriptions like (11):

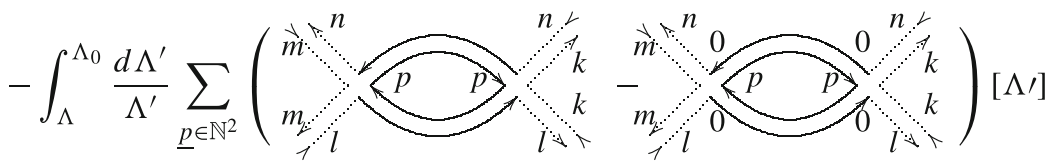

$$
\begin{aligned}
& =\int_{\Lambda}^{\Lambda_{0}} \frac{d \Lambda^{\prime}}{\Lambda^{\prime}} \int_{\Lambda^{\prime}}^{\Lambda_{0}} \frac{d \Lambda^{\prime \prime}}{\Lambda^{\prime \prime}} \sum_{\underline{p} \in \mathbb{N}^{2}}\left(\left(Q_{\underline{n} \underline{p} ; \underline{p} \underline{n}}-Q_{\underline{0} \underline{p} ; \underline{p} \underline{0}}\right)\left(\Lambda^{\prime}\right) Q_{\underline{l} \underline{p} ; \underline{p} \underline{\underline{l}}}\left(\Lambda^{\prime \prime}\right)\right. \\
& \left.+Q_{\underline{0} \underline{p} ; \underline{p} \underline{0}}\left(\Lambda^{\prime}\right)\left(Q_{\underline{l} \underline{p} ; \underline{p} \underline{\underline{l}}}-Q_{\underline{0} \underline{p} ; \underline{p} \underline{0}}\right)\left(\Lambda^{\prime \prime}\right)\right) \sim \frac{C(|\underline{n}|+|\underline{l}|)}{\theta \Omega^{2} \Lambda^{2}} .
\end{aligned}
$$

This explains the polynomial in fractions like $\frac{|\underline{m}|}{\theta \Lambda^{2}}$ in (13).

As the estimation (13) is achieved by a finite number of initial conditions at $\Lambda_{R}$ (see (11)), the noncommutative $\phi^{4}$-model with oscillator term is renormalisable to all orders in perturbation theory. These initial conditions correspond to normalisation experiments for the mass, the field amplitude, the coupling constant, and the oscillator frequency in the bare action related to (1).

\section{Vanishing of the $\beta$-Function}

Knowing the relevant/marginal couplings, we can compute Feynman graphs with sharp matrix cut-off $\mathcal{N}$. The most important question concerns the $\beta$-function appearing in the renormalisation group equation which describes the cut-off dependence of the expansion coefficients $\Gamma_{m_{1} n_{1} ; \ldots ; m_{N} n_{N}}$ of the effective action when imposing normalisation conditions for the relevant and marginal couplings. We have [8]

$\lim _{\mathcal{N} \rightarrow \infty}\left(\mathcal{N} \frac{\partial}{\partial \mathcal{N}}+N \gamma+\mu_{0}^{2} \beta_{\mu_{0}} \frac{\partial}{\partial \mu_{0}^{2}}+\beta_{\lambda} \frac{\partial}{\partial \lambda}+\beta_{\Omega} \frac{\partial}{\partial \Omega}\right) \Gamma_{\underline{m}_{1} \underline{n}_{1} ; \ldots ; \underline{m}_{N} \underline{n}_{N}}\left[\mu_{0}, \lambda, \Omega, \mathcal{N}\right]=0$,

where

$$
\begin{aligned}
\beta_{\lambda} & =\mathcal{N} \frac{\partial}{\partial \mathcal{N}}\left(\lambda\left[\mu_{R}, \lambda_{R}, \Omega_{R}, \mathcal{N}\right]\right), & \beta_{\Omega} & =\mathcal{N} \frac{\partial}{\partial \mathcal{N}}\left(\Omega\left[\mu_{R}, \lambda_{R}, \Omega_{R}, \mathcal{N}\right]\right), \\
\beta_{\mu_{0}} & =\frac{\mathcal{N}}{\mu_{0}^{2}} \frac{\partial}{\partial \mathcal{N}}\left(\mu_{0}^{2}\left[\mu_{R}, \lambda_{R}, \Omega_{R}, \mathcal{N}\right]\right), & \gamma & =\mathcal{N} \frac{\partial}{\partial \mathcal{N}}\left(\ln \mathcal{Z}\left[\mu_{R}, \lambda_{R}, \Omega_{R}, \mathcal{N}\right]\right) .
\end{aligned}
$$

Here, $\mathcal{Z}$ is the wavefunction renormalisation. To one-loop order, we find [8]

$$
\begin{array}{rlrl}
\beta_{\lambda} & =\frac{\lambda_{R}^{2}}{48 \pi^{2}} \frac{\left(1-\Omega_{R}^{2}\right)}{\left(1+\Omega_{R}^{2}\right)^{3}}, & \beta_{\Omega}=\frac{\lambda_{R} \Omega_{R}}{96 \pi^{2}} \frac{\left(1-\Omega_{R}^{2}\right)}{\left(1+\Omega_{R}^{2}\right)^{3}}, \\
\beta_{\mu_{0}}=-\frac{\lambda_{R}\left(4 \mathcal{N} \ln (2)+\frac{\left(8+\theta \mu_{R}^{2}\right) \Omega_{R}^{2}}{\left(1+\Omega_{R}^{2}\right)^{2}}\right)}{48 \pi^{2} \theta \mu_{R}^{2}\left(1+\Omega_{R}^{2}\right)}, & \gamma=\frac{\lambda_{R}}{96 \pi^{2}} \frac{\Omega_{R}^{2}}{\left(1+\Omega_{R}^{2}\right)^{3}} .
\end{array}
$$

From (18) and (19), one finds that $\frac{\lambda}{\Omega^{2}}$ remains constant under the renormalisation flow. The integration of the resulting differential equation shows that, starting from given small values for $\Omega_{R}, \lambda_{R}$ at $\mathcal{N}_{R}$, the frequency grows in a small region around $\ln \frac{\mathcal{N}}{\mathcal{N}_{R}}=\frac{48 \pi^{2}}{\lambda_{R}}$ to $\Omega \approx 1$. 
The coupling constant approaches $\lambda_{\infty}=\frac{\lambda_{R}}{\Omega_{R}^{2}}$, which can be made small for sufficiently small $\lambda_{R}$. This leaves the chance of a non-perturbative construction [23] of this model.

The key observation for all subsequent work is the following: The one-loop renormalisation flow has a non-trivial UV fixed point given by the self-dual model $\Omega=1$ in (2) where $\beta_{\Omega}=0$ to all orders. Working exactly at $\Omega=1$ leads according to (4) to the formulation as a matrix model

$$
S[\Phi]=(2 \pi \theta)^{2}\left(\frac{1}{2} \sum_{\underline{m}, \underline{n}} H_{\underline{m} \underline{n}} \Phi_{\underline{m} \underline{n}} \Phi_{\underline{n} \underline{m}}+\frac{\lambda Z^{2}}{4} \sum_{\underline{m}, \underline{n}, \underline{k}, \underline{l} \in \mathbb{N}^{2}} \Phi_{\underline{m} \underline{n}} \Phi_{\underline{n} \underline{k}} \Phi_{\underline{k} \underline{l}} \Phi_{\underline{l} \underline{m}}\right),
$$

where $H_{\underline{m} \underline{n}}=Z \frac{4}{\theta}(|\underline{m}|+|\underline{n}|)+\left(\mu_{\text {bare }}^{2}+2\right)$ and $|\underline{m}|:=m_{1}+m_{2}$. Up to an inessential factor $\frac{1}{64 \pi^{2}}$ and a shift $\mu_{\text {bare }}^{2}+2 \mapsto \mu_{\text {bare }}^{2}$, this action can also be written as

$$
S[\Phi]=V \operatorname{tr}\left(E \Phi^{2}+\frac{\lambda Z^{2}}{4} \Phi^{4}\right),
$$

where $E=\left(E_{\underline{m}} \delta_{\underline{m} \underline{n}}\right)$ with $E_{\underline{m}}=Z \frac{|\underline{m}|}{\sqrt{V}}+\frac{1}{2} \mu_{\text {bare }}^{2}$ and $V=\left(\frac{\theta}{4}\right)^{2}$. This form, closely related to models studied in [18], will be the starting point of a general treatment of such models in Section 4. Starting from (20), Disertori and Rivasseau were able to prove that the $\beta$-function vanishes to three-loop order [4]. This result led to the conjecture of $\beta=0$ to all orders, which was eventually proved in [5] by a combination of Ward identities and Schwinger-Dyson equation.

We understood immediately that the method suggested in [5] has the potential to provide an exact solution of the model. Indeed we proved in [12] that the 2-point function satisfies (in a certain limit) a closed non-linear integral equation, which is essentially equation (30) below, but expressed in other variables. In [12], we also gave a perturbative approximation of the solution. In the following years, we tried to extract non-perturbative information out of (27). We were joined by Prof. Zeidler in 2011 who tried to use techniques from non-linear functional analysis. Although a breakthrough was not achieved in this way, the exchange with Prof. Zeidler stimulated a different strategy via singular integral equations, which will be described in the next section.

\section{Exact Solution of the Quartic Matrix Model}

Adding a source term to the action (21), we define the partition function as

$$
\mathcal{Z}[J]=\int \mathcal{D}[\Phi] \exp (-S[\Phi]+V \operatorname{tr}(\Phi J))
$$

where $\mathcal{D}[\Phi]$ is the extension of the Lebesgue measure from finite-rank operators to $\mathcal{L}^{2}(H)$ and $J$ a test function matrix. For $\lambda=0$ in (21), $\frac{\mathcal{D}[\Phi]}{\mathcal{Z}[0]}$ would be the Gaussian measure of covariance determined by $E$. In the presence of interaction $\lambda \neq 0$, a rigorous construction of the measure cannot be expected. Instead we will derive, for finite matrix size $\mathcal{N}$, equations between connected correlation functions formally defined by

$$
\left\langle\varphi_{a_{1} b_{1}} \ldots \varphi_{a_{N} b_{N}}\right\rangle_{c}=\left.\frac{\partial^{N} \log \mathcal{Z}[J]}{\partial J_{b_{1} a_{1}} \ldots \partial J_{b_{N} a_{N}}}\right|_{J=0} .
$$


Then, we prove that, after renormalisation, these equations have a well-defined limit $\mathcal{N}, V \rightarrow \infty$ which is exact in $\lambda$. We are then able to reduce this problem to a fixed point problem where analytic and numerical techniques are applied.

\subsection{Ward Identity}

The first steps apply for actions of the form (21) with arbitrary positive $E$ and even general polynomial interaction $\frac{\lambda}{4} \Phi^{4} \mapsto P[\Phi]$.

Unitary operators $U$ give rise to a transformation $\Phi \mapsto \tilde{\Phi}=U \Phi U^{*}$. Since the space of selfadjoint compact operators is invariant under the adjoint action, we have

$$
\int \mathcal{D}[\Phi] \exp (-S[\Phi]+V \operatorname{tr}(\Phi J))=\int \mathcal{D}[\tilde{\Phi}] \exp (-S[\tilde{\Phi}]+V \operatorname{tr}(\tilde{\Phi} J))
$$

Unitary invariance $\mathcal{D}[\tilde{\Phi}]=\mathcal{D}[\Phi]$ of the Lebesgue measure implies

$$
0=\int \mathcal{D}[\Phi]\{\exp (-S[\Phi]+V \operatorname{tr}(\Phi J))-\exp (-S[\tilde{\Phi}]+V \operatorname{tr}(\tilde{\Phi} J))\}
$$

Note that the integrand $\{\ldots\}$ itself does not vanish because $\operatorname{tr}\left(E \Phi^{2}\right)$ and $\operatorname{tr}(\Phi J)$ are not unitary invariant; only the interaction term $\operatorname{tr}(P[\Phi])=\operatorname{tr}(P[\tilde{\Phi}])$ is invariant. Linearisation of $U$ about the identity operator leads to the Ward identity

$$
0=\int \mathcal{D}[\Phi]\{E \Phi \Phi-\Phi \Phi E-J \Phi+\Phi J\} \exp (-S[\Phi]+V \operatorname{tr}(\Phi J))
$$

We can always place ourselves in an orthonormal basis of $H$ where $E$ is diagonal (but $J$ is not). Since $E$ is of compact resolvent, $E$ has eigenvalues $E_{a}>0$ of finite multiplicity $\mu_{a}$. We thus label the matrices by an enumeration of the (necessarily discrete) eigenvalues of $E$ and an enumeration of the basis vectors of the finite-dimensional eigenspaces. Writing $\Phi$ in $\{\ldots\}$ of (23) as functional derivative $\Phi_{a b}=\frac{\partial}{V \partial J_{b a}}$, we have proved (first obtained in [5]):

Proposition 1 The partition function $\mathcal{Z}[J]$ of the matrix model defined by the external matrix $E$ satisfies the $|I| \times|I|$ Ward identities

$$
0=\sum_{n \in I}\left(\frac{\left(E_{a}-E_{p}\right)}{V} \frac{\partial^{2} \mathcal{Z}}{\partial J_{a n} \partial J_{n p}}+J_{p n} \frac{\partial \mathcal{Z}}{\partial J_{a n}}-J_{n a} \frac{\partial \mathcal{Z}}{\partial J_{n p}}\right)
$$

Without loss of generality, we can assume that the map $I \ni m \mapsto E_{m} \in \mathbb{R}_{+}$is injective. Namely, correlation functions will only depend on the set of eigenvalues $\left(E_{m}\right)$ of $E$. Partitioning the index set $I$ into equivalence classes $[m]$ which have the same $E_{m}$, the index sum over a function that only depends on $E_{m}$ becomes $\sum_{m \in I} f(m)=\sum_{[m] \in[I]} \mu_{[m]} f([m])$. Therefore, at the expense of adding a measure $\mu_{[m]}=\operatorname{dim} \operatorname{ker}\left(E-E_{m}\right.$ id $)$, we can assume that $m \mapsto E_{m}$ is injective.

Next, we turn the Ward identity (24) for injective $m \mapsto E_{m}$ into a formula for the second derivative $\sum_{n \in I} \frac{\partial^{2} \mathcal{Z}[J]}{\partial J_{a n} \partial J_{n p}}$ of the partition function. Thereby, a term proportional to $\delta_{a p}$ arises which was identified in [14, Theorem 2.3]. 


\subsection{Schwinger-Dyson Equations}

We can write the action as $S=\frac{V}{2} \sum_{a, b}\left(E_{a}+E_{b}\right) \Phi_{a b} \Phi_{b a}+V S_{i n t}[\Phi]$, where $E_{a}$ are the eigenvalues of $E$. Functional integration yields, up to an irrelevant constant,

$$
\mathcal{Z}[J]=e^{-V S_{\text {int }}\left[\frac{\partial}{V \partial J}\right]} e^{\frac{V}{2}\langle J, J\rangle_{E}}, \quad\langle J, J\rangle_{E}:=\sum_{m, n \in I} \frac{J_{m n} J_{n m}}{E_{m}+E_{n}} .
$$

Instead of a perturbative expansion of $e^{-V S_{i n t}\left[\frac{\partial}{V \partial J}\right]}$, we apply those $J$-derivatives to (25) which give rise to a correlation function $G_{\ldots}$ on the left-hand side. On the right-hand side of (25), these external derivatives combine with internal derivatives from $S_{i n t}\left[\frac{\partial}{V \partial J}\right]$ to certain identities for $G_{\ldots . .}$. These Schwinger-Dyson equations are often of little use because they express an $N$-point function in terms of $(N+2)$-point functions.

In the field-theoretical matrix models under consideration, the Ward identity lets this tower of Schwinger-Dyson equation collapse. We prove in [14]:

Proposition 2 The 2-point function of a quartic matrix model with action $S=V \operatorname{tr}\left(E \Phi^{2}+\right.$ $\left.\frac{\lambda}{4} \Phi^{4}\right)$ satisfies for injective $m \mapsto E_{m}$ the Schwinger-Dyson equation

$$
\left.\begin{array}{rl}
G_{|a b|}= & \frac{1}{E_{a}+E_{b}}-\frac{\lambda}{E_{a}+E_{b}} \frac{1}{V} \sum_{p \in I}\left(G_{|a b|} G_{|a p|}-\frac{G_{|p b|}-G_{|a b|}}{E_{p}-E_{a}}\right) \\
& -\frac{\lambda}{V^{2}\left(E_{a}+E_{b}\right)}\left(\begin{array}{c}
G_{|a| a \mid} G_{|a b|}+\frac{1}{V} \sum_{n \in I} G_{|a n| a b \mid} \\
\left.+G_{|a a a b|}+G_{|b a b a|}-\frac{G_{|b| b \mid}-G_{|a| b \mid}}{E_{b}-E_{a}}\right)
\end{array}\right\} \\
& -\frac{\lambda}{V^{4}\left(E_{a}+E_{b}\right)} G_{|a| a|a b|} .
\end{array}\right\}
$$

It can be checked [14] that in a genus expansion $G_{\ldots}=\sum_{g=0}^{\infty} V^{-2 g} G_{\ldots}^{(g)}$ (which is probably not convergent but Borel summable), precisely the line (26a) preserves the genus, the lines (26b) increase $g \mapsto g+1$ and the line (26c) increases $g \mapsto g+2$. In particular, in a scaling limit $V \rightarrow \infty$ with $\frac{1}{V} \sum_{p \in I}$ finite, the exact Schwinger-Dyson equation for $G_{|a b|}$ coincides with its restriction (26a) to the planar sector $g=0$, a closed non-linear equation for $G_{|a b|}^{(0)}$ alone:

$$
G_{|a b|}^{(0)}=\frac{1}{E_{a}+E_{b}}-\frac{\lambda}{E_{a}+E_{b}} \frac{1}{V} \sum_{p \in I}\left(G_{|a b|}^{(0)} G_{|a p|}^{(0)}-\frac{G_{|p b|}^{(0)}-G_{|a b|}^{(0)}}{E_{p}-E_{a}}\right) .
$$

We have derived in 2007/2008 this self-consistency equation for the Moyal model by the graphical method proposed by [5]. In this form, (27) is meaningless because $\sum_{p \in I}$ diverges. In 2009 we solved the renormalisation problem, namely the renormalisation of infinitely many Feynman graphs at once [12]. This renormalisation increases the non-linearity. In [12] we have solved (27) perturbatively to $\mathcal{O}\left(\lambda^{3}\right)$. After several years of setbacks with the non-perturbative solution, a breakthrough came in 2012: The equation (27) can be turned into an equation (given in (32) below) which is linear in the difference $G_{|a b|}^{(0)}-G_{|a 0|}^{(0)}$ to the boundary and non-linear only in $G_{|a 0|}^{(0)}$ ! 
It turns out that a real theory with $\Phi=\Phi^{*}$ admits a short-cut which directly gives the higher $N$-point functions without any index summation. Since the equations for $G_{\ldots}$ are real and $\overline{J_{a b}}=J_{b a}$, the reality $\mathcal{Z}=\overline{\mathcal{Z}}$ implies (in addition to invariance under cyclic permutations) invariance under orientation reversal

$$
G_{\left|p_{0}^{1} p_{1}^{1} \ldots p_{N_{1}-1}^{1}\right| \ldots\left|p_{0}^{B} p_{1}^{B} \ldots p_{N_{B}-1}^{B}\right|}=G_{\left|p_{0}^{1} p_{N_{1}-1}^{1} \ldots p_{1}^{1}\right| \ldots\left|p_{0}^{B} p_{N_{B}-1}^{B} \ldots p_{1}^{B}\right|}
$$

Whereas empty for $G_{|a b|}$, in $\left(E_{a}+E_{b_{1}}\right) G_{a b_{1} b_{2} \ldots b_{N-1}}-\left(E_{a}+E_{b_{N-1}}\right) G_{a b_{N-1} \ldots b_{2} b_{1}}$ the identities (28) lead to many cancellations which result in a universal algebraic recursion formula:

\section{Proposition 3}

$$
\begin{aligned}
G_{\left|b_{0} b_{1} \ldots b_{N-1}\right|}= & (-\lambda) \sum_{l=1}^{\frac{N-2}{2}} \frac{G_{\left|b_{0} b_{1} \ldots b_{2 l-1}\right|} G_{\left|b_{2 l} b_{2 l+1} \ldots b_{N-1}\right|}-G_{\left|b_{2 l} b_{1} \ldots b_{2 l-1}\right|} G_{\left|b_{0} b_{2 l+1} \ldots b_{N-1}\right|}}{\left(E_{b_{0}}-E_{b_{2 l}}\right)\left(E_{b_{1}}-E_{b_{N-1}}\right)} \\
& +\frac{(-\lambda)}{V} \sum_{k=1}^{N-1} \frac{G_{\left|b_{0} b_{1} \ldots b_{k-1}\right| b_{k} b_{k+1} \ldots b_{N-1} \mid}-G_{\left|b_{k} b_{1} \ldots b_{k-1}\right| b_{0} b_{k+1} \ldots b_{N-1} \mid}}{\left(E_{b_{0}}-E_{b_{k}}\right)\left(E_{b_{1}}-E_{b_{N-1}}\right)}
\end{aligned}
$$

The last line of (29) increases the genus and is absent in $G_{\left|b_{0} b_{1} \ldots b_{N-1}\right|}^{(0)}$.

We make the following key observation: An affine transformation $E \mapsto Z E+C$ together with a corresponding rescaling $\lambda \mapsto Z^{2} \lambda$ leaves the algebraic (29) invariant:

Theorem 2 Given a real quartic matrix model with $S=V \operatorname{tr}\left(E \Phi^{2}+\frac{\lambda}{4} \Phi^{4}\right)$ and $m \mapsto E_{m}$ injective, which determines the set $G_{\left|p_{1}^{1} \ldots p_{N_{1}}^{1}\right| \ldots\left|p_{1}^{B} \ldots p_{N_{B}}^{B}\right|}$ of $\left(N_{1}+\cdots+N_{B}\right)$-point functions. Assume that the basic functions with all $N_{i} \leq 2$ are turned finite by $E_{a} \mapsto Z\left(E_{a}+\frac{\mu^{2}}{2}-\right.$ $\left.\frac{\mu_{\text {bare }}^{2}}{2}\right)$ and $\lambda \mapsto Z^{2} \lambda$. Then all functions with one $N_{i} \geq 3$

1. are finite without further need of a renormalisation of $\lambda$, i.e., all renormalisable quartic matrix models have vanishing $\beta$-function,

2. are given by universal algebraic recursion formulae in terms of renormalised basic functions with $N_{i} \leq 2$.

The theorem tells us that vanishing of the $\beta$-function for the self-dual $\Phi_{4}^{4}$-model on Moyal space (proved in [5] to all orders in perturbation theory) is generic to all quartic matrix models, and the result even holds non-perturbatively!

The universal recursion formula (29) computes the planar $N$-point function $G_{\left|b_{0} \ldots b_{N-1}\right|}$ at $B=1$ as a sum of fractions with products of 2-point functions in the numerator and products of differences of eigenvalues of $E$ in the denominator. This structure admits an interesting graphical interpretation. We draw the indices $b_{0}, \ldots b_{N-1}$ in cyclic order on the circle $S^{1}$ and represent a factor $G_{b_{i} b_{j}}$ as a chord connecting $b_{i}$ with $b_{j}$ and a factor $\frac{1}{E_{b_{i}}-E_{b_{j}}}$ as an arrow from $b_{i}$ to $b_{j}$.

The chords form the non-crossing chord diagrams counted by the Catalan number $C_{\frac{N}{2}}=\frac{N !}{\left(\frac{N}{2}+1\right) ! \frac{N}{2} !}$. 


\subsection{Renormalisation and Integral Representation}

We return to the noncommutative $\phi^{4}$-model at self-duality point $\Omega=1$, where the action is given by Eq. (20). Our general results on quartic matrix models imply that the planar 2-point function $G_{|\underline{\mid a b}|}^{(0)}$ satisfies the self-consistency equation (27),

$$
G_{|\underline{a b}|}^{(0)}=\frac{1}{E_{\underline{a}}+E_{\underline{b}}}-\frac{Z^{2} \lambda}{E_{\underline{a}}+E_{\underline{b}}} \frac{1}{V} \sum_{\underline{p} \in \mathbb{N}_{\mathcal{N}}^{2}}\left(G_{\mid \underline{|\underline{b}|}}^{(0)} G_{|\underline{\mid \underline{a} p}|}^{(0)}-\frac{G_{|\underline{p} \underline{b}|}^{(0)}-G_{|\underline{a b}|}^{(0)}}{E_{\underline{p}}-E_{\underline{a}}}\right),
$$

where we recall $E_{\underline{m}}=Z\left(\frac{|\underline{m}|}{\sqrt{V}}+\frac{\mu_{\text {bare }}^{2}}{2}\right)$. We have introduced a cut-off $\mathbb{N}_{\mathcal{N}}^{2}$ in the matrix size; the index sum diverges for $\mathbb{N}_{\mathcal{N}}^{2} \mapsto \mathbb{N}^{2}$. As usual, the renormalisation strategy consists in adjusting $Z$, $\mu_{\text {bare }}$ in such a way that the limit $\mathbb{N}_{\mathcal{N}}^{2} \mapsto \mathbb{N}^{2}$ exists. This will be achieved by normalisation conditions for the 1PI function $\Gamma_{\underline{a b}}$ defined by $G_{|a b|}^{(0)}=:\left(H_{\underline{a b}}-\Gamma_{\underline{a b}}\right)^{-1}$, where $H_{\underline{a b}}:=E_{\underline{a}}+E_{\underline{b}}$. We express (30) in terms of $\Gamma_{\underline{a b}}$ and write $\Gamma_{\underline{a b}}$ as first-order Taylor formula with remainder $\Gamma_{\underline{a b}}^{r e n}$,

$$
\Gamma_{\underline{a b}}=Z \mu_{\text {bare }}^{2}-\mu^{2}+\frac{(Z-1)}{\sqrt{V}}(|\underline{a}|+|\underline{b}|)+\Gamma_{\underline{a b}}^{r e n}, \quad \Gamma_{\underline{00}}^{r e n}=0, \quad\left(\partial \Gamma^{r e n}\right)_{\underline{00}}=0 .
$$

Equation (30) for $\Gamma_{\underline{a b}}\left[\Gamma_{\underline{a b}}^{r e n}, \mu_{b a r e}^{2}, Z\right]$ together with $\Gamma_{\underline{00}}^{r e n}=0$ and $\left(\partial \Gamma^{r e n}\right)_{\underline{00}}$ constitute three equations to determine the three functions $\Gamma_{a b}^{r e n}, \mu_{\text {bare }}^{2}, Z$. Eliminating $\mu_{\text {bare }}^{2}, Z$ thus gives rise to a closed equation for the renormalised function $\Gamma_{\underline{a b}}^{r e n}$ alone. For this elimination, it is important to note that the equations for $\Gamma_{a b}^{r e n}, \mu_{b a r e}^{2}, Z$ depend on $\underline{a}, \underline{b}$ only via the norms $|\underline{a}|,|\underline{b}|$ which parametrise the spectrum of $E$. Therefore, $\Gamma_{\underline{a b}}$ is actually a function only of $|\underline{a}|,|\underline{b}|$, and consequently the index sum reduces to $\sum_{\underline{p} \in \mathbb{N}_{\mathcal{N}}^{2}} f(|\underline{p}|)=$ $\sum_{|\underline{p}|=0}^{\mathcal{N}}(|\underline{p}|+1) f(|\underline{p}|)$.

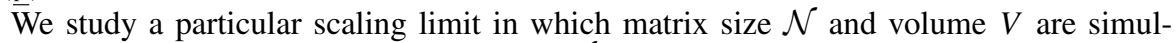
taneously sent to $\infty$ such that the ratio $\frac{\mathcal{N}}{\sqrt{V \mu^{4}}}=\Lambda^{2}(1+\mathcal{Y})$ is kept fixed. Note that $V=\left(\frac{\theta}{4}\right)^{2} \rightarrow \infty$ is a limit of extreme noncommutativity! The new parameter $(1+\mathcal{Y})$ corresponds to a finite wavefunction renormalisation, identified later to decouple our equations. The parameter $\Lambda^{2}$ represents an ultraviolet cut-off which is sent to $\Lambda \rightarrow \infty$ in the very end (continuum limit). In the scaling limit, functions of $\frac{|p|}{\sqrt{V}}=: \mu^{2}(1+\mathcal{Y}) p$ converge to functions of 'continuous matrix indices' $p \in\left[0, \Lambda^{2}\right]$. In the same way, $\Gamma_{\underline{a}}^{r e n} \underline{b}$ converges to a function $\mu^{2} \Gamma_{a b}$ with $a, b \in\left[0, \Lambda^{2}\right]$, and the discrete sum converges to a Riemann integral

$$
\frac{1}{V} \sum_{|\underline{p}|=0}^{\mathcal{N}}(|\underline{p}|+1) f\left(\frac{|\underline{p}|}{\sqrt{V}}\right) \longrightarrow \mu^{4}(1+\mathcal{Y})^{2} \int_{0}^{\Lambda^{2}} p d p f\left(\mu^{2}(1+\mathcal{Y}) p\right) .
$$

This limit makes the restriction to the planar sector (27) of (26a)-(26c) exact.

Applying $\left.\frac{d}{d b}\right|_{a=b=0}$ we get $Z$ in terms of $\Gamma_{a b}$ (and its derivative). Inserted back one gets a highly non-linear integro-differential equation. We can reduce the non-linearity by subtracting from it the same equation taken at $b=0$. In terms of $G_{a b}:=$ $\left((a+b)(1+\mathcal{Y})+1-\Gamma_{a b}\right)^{-1}$, this difference equation reads

$$
\frac{Z^{-1}}{(1+\mathcal{Y})}\left(\frac{1}{G_{a b}}-\frac{1}{G_{a 0}}\right)=b-\lambda \int_{0}^{\Lambda^{2}} p d p \frac{\frac{G_{p b}}{G_{a b}}-\frac{G_{p 0}}{G_{a 0}}}{p-a} .
$$


Differentiation $\left.\frac{d}{d b}\right|_{a=b=0}$ of (31) yields $Z$ in terms of $G_{a b}$ and its derivative. The resulting derivative $G^{\prime}$ can be avoided by adjusting

$$
\mathcal{Y}:=-\lambda \lim _{b \rightarrow 0} \int_{0}^{\Lambda^{2}} d p \frac{G_{p b}-G_{p 0}}{b} .
$$

This choice leads to $\frac{Z^{-1}}{(1+\mathcal{Y})}=1-\lambda \int_{0}^{\Lambda^{2}} d p G_{p 0}$, which is a perturbatively divergent integral for $\Lambda \rightarrow \infty$. Inserting $Z^{-1}$ and $\mathcal{Y}$ back into (31) we end up in a linear integral equation for the difference function $D_{a b}:=\frac{a}{b}\left(G_{a b}-G_{a 0}\right)$ to the boundary:

$$
\left(\frac{b}{a}+\frac{1}{a G_{a 0}}\right) D_{a b}+G_{a 0}=\lambda \int_{0}^{\Lambda^{2}} d p\left(\frac{D_{p b}-D_{a b} \frac{G_{p 0}}{G_{a 0}}}{p-a}\right) .
$$

The non-linearity restricts to the boundary function $G_{a 0}$ where the second index is put to zero. Assuming $a \mapsto G_{a b}$ Hölder-continuous, we can pass to Cauchy principal values. In terms of the finite Hilbert transform

$$
\mathcal{H}_{a}^{\Lambda}[f(\bullet)]:=\frac{1}{\pi} \lim _{\epsilon \rightarrow 0}\left(\int_{0}^{a-\epsilon}+\int_{a+\epsilon}^{\Lambda^{2}}\right) \frac{f(q) d q}{q-a},
$$

the integral equation (32) becomes

$$
\left(\frac{b}{a}+\frac{1+\lambda \pi a \mathcal{H}_{a}^{\Lambda}\left[G_{\bullet}\right]}{a G_{a 0}}\right) D_{a b}-\lambda \pi \mathcal{H}_{a}^{\Lambda}\left[D_{\bullet b}\right]=-G_{a 0} .
$$

\subsection{The Carleman Solution}

Equation (34) is a well-known singular integral equation of Carleman type [3, 24]:

Theorem 3 ([24], transformed from $[-1,1]$ to $\left.\left[0, \Lambda^{2}\right]\right)$ The singular linear integral equation

$$
\left.h(a) y(a)-\lambda \pi \mathcal{H}_{a}^{\Lambda}[y]=f(a), \quad a \in\right] 0, \Lambda^{2}[,
$$

is for $h(a)$ continuous on $] 0, \Lambda^{2}\left[\right.$, Hölder-continuous near $0, \Lambda^{2}$, and $f \in L^{p}$ for some $p>1$ (determined by $\vartheta(0)$ and $\left.\vartheta\left(\Lambda^{2}\right)\right)$ solved by

$$
\begin{aligned}
& y(a)=\frac{\sin (\vartheta(a)) e^{-\mathcal{H}_{a}^{\Lambda}[\pi-\vartheta]}}{\lambda \pi a}\left(a f(a) e^{\mathcal{H}_{a}^{\Lambda}[\pi-\vartheta]} \cos (\vartheta(a))\right. \\
&\left.+\mathcal{H}_{a}^{\Lambda}\left[e^{\mathcal{H}_{\bullet}^{\Lambda}[\pi-\vartheta]} \bullet f(\bullet) \sin (\vartheta(\bullet))\right]+C\right) \\
& \stackrel{*}{=} \frac{\sin (\vartheta(a)) e^{\mathcal{H}_{a}^{\Lambda}[\vartheta]}}{\lambda \pi}\left(f(a) e^{-\mathcal{H}_{a}^{\Lambda}[\vartheta]} \cos (\vartheta(a))\right. \\
&\left.+\mathcal{H}_{a}^{\Lambda}\left[e^{-\mathcal{H}_{\bullet}^{\Lambda}[\vartheta]} f(\bullet) \sin (\vartheta(\bullet))\right]+\frac{C^{\prime}}{\Lambda^{2}-a}\right),
\end{aligned}
$$

where $\vartheta(a)=\arctan _{[0, \pi]}\left(\frac{\lambda \pi}{h(a)}\right), \sin (\vartheta(a))=\frac{|\lambda \pi|}{\sqrt{(h(a))^{2}+(\lambda \pi)^{2}}} \geq 0$ and $C, C^{\prime}$ are arbitrary constants. 
The possibility of $C, C^{\prime} \neq 0$ is due to the fact that the finite Hilbert transform has a kernel, in contrast to the infinite Hilbert transform with integration over $\mathbb{R}$. The two formulae (35a) and (35b) are formally equivalent, but the solutions belong to different function classes and normalisation conditions may (and will) make a choice.

From (34), one introduces an angle function, which leads to a representation first for $G_{a 0}$ :

Lemma 1 The angle function $\tau_{b}(a):=\arctan _{[0, \pi]}\left(\frac{|\lambda| \pi a}{b+\frac{1+\lambda \pi a \mathcal{H}_{a}^{\Lambda}\left[G_{\bullet 0}\right]}{G_{a 0}}}\right)$ is for $b=0$ reverted to

$$
G_{a 0}=\frac{\sin \left(\tau_{0}(a)\right)}{|\lambda| \pi a} \mathrm{e}^{\operatorname{sign}(\lambda)\left(\mathcal{H}_{0}^{\Lambda}\left[\tau_{0}(\bullet)\right]-\mathcal{H}_{a}^{\Lambda}\left[\tau_{0}(\bullet)\right]\right)} \begin{cases}1 & \text { for } \lambda<0, \\ \left(1+\frac{C a}{\Lambda^{2}-a}\right) & \text { for } \lambda>0,\end{cases}
$$

where $C$ is an arbitrary constant.

Recall that $G_{a 0}$ forms the inhomogeneity in the Carleman equation (34). We insert (36) into the Carleman solution (35) for (34) and obtain with the addition theorem $|\lambda| \pi a \sin \left(\tau_{d}(a)-\tau_{b}(a)\right)=(b-d) \sin \tau_{b}(a) \sin \tau_{d}(a)$ after essentially the same steps as in the proof of (36):

Theorem 4 ([15]) The full matrix 2-point function $G_{a b}$ of self-dual $\phi_{4}^{4}$-theory on Moyal space is in the limit $\theta \rightarrow \infty$ given in terms of the boundary 2-point function $G_{a 0}$ by the equation

$$
G_{a b}=\frac{\sin \left(\tau_{b}(a)\right)}{|\lambda| \pi a} \mathrm{e}^{\operatorname{sign}(\lambda)\left(\mathcal{H}_{0}^{\Lambda}\left[\tau_{0}(\bullet)\right]-\mathcal{H}_{a}^{\Lambda}\left[\tau_{b}(\bullet)\right]\right)} \begin{cases}1 & \text { for } \lambda<0, \\ \left(1+\frac{C a+b F(b)}{\Lambda^{2}-a}\right) & \text { for } \lambda>0,\end{cases}
$$

where $C$ is an undetermined constant and $b F(b)$ an undetermined function of $b$ vanishing at $b=0$.

Some remarks:

- We have proved this theorem in [14] for $\lambda>0$ under the assumption $C^{\prime}=0$ in (35b), but knew that non-trivial solutions of the homogeneous Carleman equation parametrised by $C^{\prime} \neq 0$ are possible. That no such term arises for $\lambda<0$ (if angles are redefined $\vartheta \mapsto \tau$ ) was proved in [15].

- We expect $C, F$ to be $\Lambda$-dependent so that $\left(1+\frac{C a+b F(b)}{\Lambda^{2}-a}\right) \stackrel{\Lambda \rightarrow \infty}{\longrightarrow} 1+\tilde{C} a+b \tilde{F}(b)$.

- An important observation is $G_{a b} \geq 0$, at least for $\lambda<0$. This is a truly non-perturbative result; individual Feynman graphs show no positivity at all!

- As in [12], the equation for $G_{a b}$ can be solved perturbatively. Matching at $\lambda=0$ requires $C, F$ to be flat functions of $\lambda$ (all derivatives vanish at zero). Because of $\mathcal{H}_{a}^{\Lambda}\left[G_{\bullet 0} \stackrel{a \rightarrow \Lambda^{2}}{\longrightarrow}-\infty\right.$, the naïve arctan series is dangerous for $\lambda>0$. Unless there are cancellations, we expect zero radius of convergence!

- From (37), we deduce the finite wavefunction renormalisation

$$
\mathcal{Y}:=-1-\left.\frac{d G_{a b}}{d b}\right|_{a=b=0}=\int_{0}^{\Lambda^{2}} \frac{d p}{(\lambda \pi p)^{2}+\left(\frac{1+\lambda \pi p \mathcal{H}_{p}^{\Lambda}\left[G_{\bullet} 0\right.}{G_{p 0}}\right)^{2}}- \begin{cases}0 & \text { for } \lambda<0, \\ F(0) & \text { for } \lambda>0 .\end{cases}
$$


- The partition function $\mathcal{Z}$ is undefined for $\lambda<0$. But the Schwinger-Dyson equations for $G_{a b}$ and for higher functions, and with them $\log \mathcal{Z}$, extend to $\lambda<0$. These extensions are unique but probably not analytic in a neighbourhood of $\lambda=0$.

Given the boundary function $G_{a 0}$, the Carleman theory computes the full 2-point function $G_{a b}$ via (37). In particular, we get $G_{0 b}$ as function of $G_{a 0}$. But the 2-point function is symmetric, $G_{a b}=G_{b a}$, and the special case $b=0$ leads to the following self-consistency equation:

Proposition 4 The limit $\theta \rightarrow \infty$ of $\phi_{4}^{4}$-theory on Moyal space is determined by the solution of the fixed point equation $G=T G$,

$$
G_{b 0}=\frac{\left\{\begin{array}{ll}
1 & \text { for } \lambda<0, \\
1+b F(b) & \text { for } \lambda>0
\end{array}\right\}}{1+b} \exp \left(-\lambda \int_{0}^{b} d t \int_{0}^{\Lambda^{2}} \frac{d p}{(\lambda \pi p)^{2}+\left(t+\frac{1+\lambda \pi p \mathcal{H}_{p}^{\Lambda}\left[G_{\bullet} 0\right]}{G_{p 0}}\right)^{2}}\right) .
$$

At this point, we can eventually send $\Lambda \rightarrow \infty$.

In [14], we proved via the Schauder fixed point theorem that (39) has a (smooth) solution for $\lambda>0$ (assuming $F(b)=0$ ) bounded by $0 \leq G_{b 0} \leq \frac{1}{1+b}$. For the much more complicated case $\lambda<0$, we proved in our contribution [16] to the 75th birthday of Prof. Zeidler the following result:

Theorem 5 Let $-\frac{1}{6} \leq \lambda \leq 0$. Then, (39) has a $C_{0}^{1}$-solution

$$
\frac{1}{(1+b)^{1-|\lambda|}} \leq G_{0 b} \leq \frac{1}{(1+b)^{1-\frac{|\lambda|}{1-2|\lambda|}}} .
$$

In [15], we solved (39) numerically by approximating $G_{0 b}$ as a piecewise linear function on $\left[0, \Lambda^{2}\right]$ sampled according to a geometric progression and by viewing (39) as iteration $G_{0 b}^{i+1}=\left(T G^{i}\right)_{0 b}$ for some initial function $G^{0}$. We confirmed the convergence of this iteration in Lipschitz norm for a large range $\lambda \in \mathbb{R}$ of either sign. It turned out that the required symmetry $G_{a b}=G_{b a}$ does not hold for $\lambda>0$, which is a clear hint that $F(b) \neq 0$ for $\lambda>0$. For $\lambda<0$ everything is consistent within small numerical errors. From the solution of (39) we get $G_{a b}$ via (37) and then all higher correlation functions via the universal algebraic recursion formulae. For $\lambda<0$, all these quantities of the model can be obtained with sufficient precision.

We find clear evidence in [15] for a second-order phase transition at $\lambda_{c} \approx-0.39$, which is a common critical value in several independent problems. The most obvious signal is a plot of the derivative $1+\mathcal{Y}:=-\left.\frac{d G_{0 b}}{d b}\right|_{b=0}$ as function of $\lambda$ shown in Fig. 1.

Globally, we found that the numeric solution is close to (but not exactly) a power law $G_{b 0} \propto \frac{1}{(1+b)^{1-\eta(\lambda) / 2}}$, where $\eta$ has opposite sign as $\lambda$. This numerical conjecture was later made precise and proved in [16]. We have indications that the exact critical coupling constant will be $\lambda_{c}=-\frac{1}{\pi}$.

We discuss in the next section how the sign on $\eta$ relates to reflection positivity of Schwinger functions made from $G_{\ldots . . .}$. Reflection positivity requires $\eta \geq 0$ which excludes (unless $F(b) \neq 0$ reverses the behaviour) the stable case $\lambda>0$ and prefers $\lambda \leq 0$ where the partition function is meaningless. 


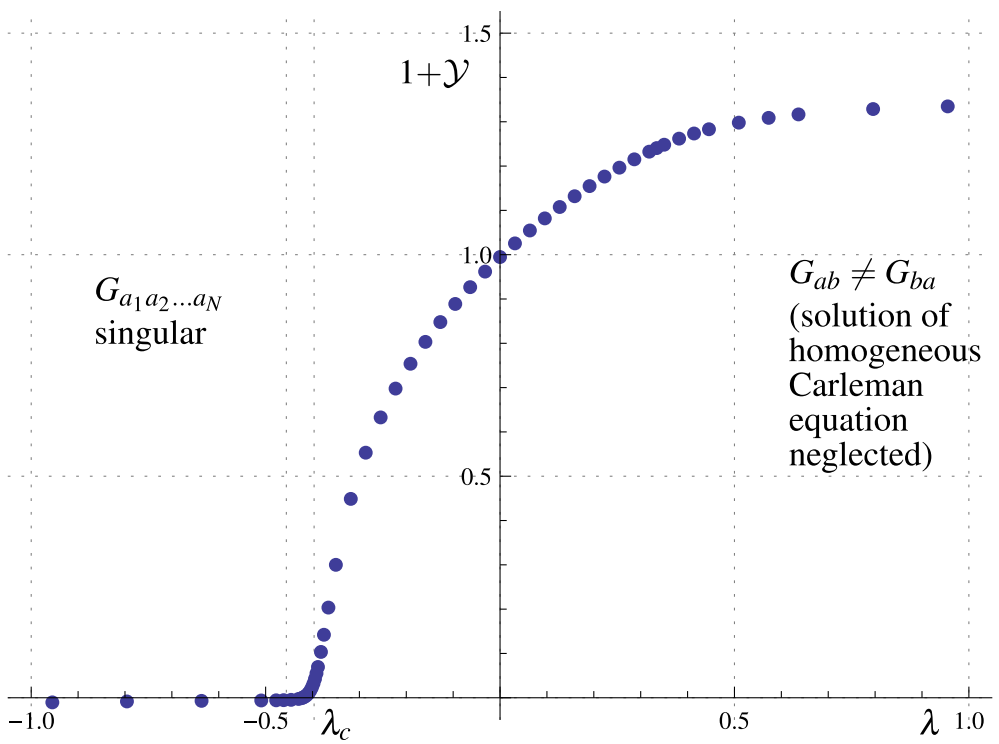

Fig. $11+\mathcal{Y}:=-\left.\frac{d G_{0 b}}{d b}\right|_{b=0}$ as function of $\lambda$, based on $G_{0 b}$ computed for $\Lambda^{2}=10^{7}$ with $L=2000$ sample points

\section{Schwinger Functions and Reflection Positivity}

\subsection{Reverting the Matrix Basis}

We are interested here in the limit to Schwinger functions in position space. For this end, we revert the matrix representation and take the infinite volume limit $V \mu^{4} \rightarrow \infty$, where we carefully have to pass to densities. Absolute position $x \in \mathbb{R}^{4}$ have no meaning, only $\mu x$ can be used. This means that we consider

$$
\left\langle\phi\left(\mu x_{1}\right) \ldots \phi\left(\mu x_{N}\right)\right\rangle \equiv \sum_{\underline{m}_{1}, \underline{n}_{1}, \ldots, \underline{m}_{N}, \underline{n}_{N} \in \mathbb{N}^{2}} f_{\underline{m}_{1} \underline{m}_{2}}\left(\mu x_{1}\right) \cdots f_{\underline{m}_{N} \underline{m}_{N}}\left(\mu x_{N}\right)\left\langle\varphi_{\underline{m}_{1} \underline{n}_{1}} \ldots \varphi_{\underline{m}_{N} \underline{n}_{N}}\right\rangle,
$$

where the matrix correlation functions are formally given by (22) and the $f_{m \underline{n}}$ by (3) and subsequent equations. More precisely we define:

Definition 1 The connected Schwinger functions associated with the action (20) are

$$
\begin{aligned}
& \mu^{N} S_{c}\left(\mu x_{1}, \ldots, \mu x_{N}\right) \\
& :=\left.\lim _{V \mu^{4} \rightarrow \infty} \sum_{\underline{m}_{1}, \underline{n}_{1}, \ldots, \underline{m}_{N}, \underline{n}_{N} \in \mathbb{N}^{2}} f_{\underline{m}_{1} \underline{n}_{1}}\left(\mu x_{1}\right) \cdots f_{\underline{m}_{N} \underline{n}_{N}}\left(\mu x_{N}\right) \frac{\mu^{4 N} \partial^{N} \mathcal{F}[J]}{\partial J_{\underline{m}_{1} \underline{n}_{1}} \ldots \partial J_{\underline{m}_{N} \underline{n}_{N}}}\right|_{J=0}, \\
& \mathcal{F}[J]:=\frac{1}{64 \pi^{2} V^{2} \mu^{8}} \log \left(\frac{\int \mathcal{D}[\Phi] e^{-S[\Phi]+V \sum_{\underline{a}, \underline{b} \in \mathbb{N}^{2}} \Phi_{\underline{a}} \underline{J_{b a}}}}{\int \mathcal{D}[\Phi] e^{-S[\Phi]}}\right)_{\substack{Z \mu_{\text {barre }}^{2} \mapsto \mu^{2} \\
Z \mapsto(1+\mathcal{Y})}} . \\
& .
\end{aligned}
$$

By ( $)_{\substack{Z \mu_{b a r e}^{2} \rightarrow \mu^{2} \\ Z \mapsto(1+\mathcal{Y})}}$, we symbolise the renormalisation of Section 4.3. 
The main question is whether these Schwinger functions satisfy the OsterwalderSchrader axioms $[20,21]$ which would allow to analytically continue the model to a true Wightman quantum field theory. The first step consists in an explicit evaluation of (40). In [13], we proved the following result:

Theorem 6 The connected $N$-point Schwinger functions of the $\phi_{4}^{4}$-model on extreme Moyal space $\theta \rightarrow \infty$ are given by

$$
\begin{aligned}
& S_{c}\left(\mu x_{1}, \ldots, \mu x_{N}\right) \\
& =\frac{1}{64 \pi^{2}} \sum_{\substack{N_{1}+\ldots+N_{B}=N \\
N_{\beta} \text { even }}} \sum_{\sigma \in \mathcal{S}_{N}}\left(\prod_{\beta=1}^{B} \frac{4^{N_{\beta}}}{N_{\beta}} \int_{\mathbb{R}^{4}} \frac{d p_{\beta}}{4 \pi^{2} \mu^{4}} e^{\left.\mathrm{i}\left\langle\frac{p_{\beta}}{\mu}, \sum_{i=1}^{N_{\beta}}(-1)^{i-1} \mu x_{\sigma\left(N_{1}+\ldots+N_{\beta-1}+i\right)}\right\rangle\right)}\right. \\
& \times G_{N_{1}}|\underbrace{\frac{\left\|p_{1}\right\|^{2}}{2 \mu^{2}(1+\mathcal{Y})}, \ldots, \frac{\left\|p_{1}\right\|^{2}}{2 \mu^{2}(1+\mathcal{Y})} \mid \cdots}_{N_{B}}| \underbrace{\frac{\left\|p_{B}\right\|^{2}}{2 \mu^{2}(1+\mathcal{Y})}, \ldots, \frac{\left\|p_{B}\right\|^{2}}{2 \mu^{2}(1+\mathcal{Y})} \mid} \cdot
\end{aligned}
$$

Some comments:

- Only a restricted sector of the underlying matrix model contributes to position space: All strands of the same boundary component carry the same matrix index.

- Schwinger functions are symmetric and invariant under the full Euclidean group. This comes truly surprising since $\theta \neq 0$ breaks both translation invariance and manifest rotation invariance. The limit $\theta \rightarrow \infty$ was expected to make this symmetry violation even worse!

- The most interesting sector is the case where every boundary component has $N_{\beta}=2$ indices. It is described by the $(2+\cdots+2)$-point functions $G\left|\frac{\left\|p_{1}\right\|^{2}}{2 \mu^{2}(1+\mathcal{Y})} \frac{\left\|p_{1}\right\|^{2}}{2 \mu^{2}(1+\mathcal{Y})}\right| \ldots\left|\frac{\left\|p_{B}\right\|^{2}}{2 \mu^{2}(1+\mathcal{Y})} \frac{\left\|p_{B}\right\|^{2}}{2 \mu^{2}(1+\mathcal{Y})}\right| \cdot$

- This sector describes the propagation and interaction of $B$ particles without any momentum exchange. This is acceptable for a $2 \mathrm{D}$ model. In four dimensions, the absence of momentum transfer is a sign of triviality.

- However, typical triviality proofs rely on clustering, analyticity in Mandelstam representation or absence of bound states. All this needs verification.

It is already clear that clustering is maximally violated. Looking for instance at the $(2+$ 2)-sector, we have

$$
\begin{aligned}
& \lim _{\mu a \rightarrow \infty} S_{c}^{2+2}\left(\mu x_{1}, \mu x_{2}, \mu\left(x_{3}+a\right), \mu\left(x_{4}+a\right)\right) \\
& \quad=\int \frac{d p d q}{4 \pi^{6} \mu^{4}} G_{\frac{\|p\|^{2}}{2 \mu^{2}(1+\mathcal{Y})}} \frac{\|p\|^{2}}{2 \mu^{2}(1+\mathcal{Y})}\left|\frac{\|q\|^{2}}{2 \mu^{2}(1+\mathcal{Y})} \frac{\|q\|^{2}}{2 \mu^{2}(1+\mathcal{Y})}\right|^{e^{\mathrm{i}\left\langle p, x_{1}-x_{2}\right\rangle+\mathrm{i}\left\langle q, x_{3}-x_{4}\right\rangle}}
\end{aligned}
$$

independent of the distance between $\left\{x_{1}, x_{2}\right\}$ on the one hand and $\left\{x_{3}, x_{4}\right\}$ on the other hand. The absence of clustering means that the state that we constructed is a mixed state. States can always be decomposed into pure states.

\subsection{Reflection Positivity}

Reflection positivity is the most decisive Osterwalder-Schrader axiom. It gives the spectrum condition and positivity of the reconstructed Hilbert space of the Minkowski model [20, 
21]. This guarantees a representation as a Laplace transform in the time component of the coordinate $\xi^{0}$, hence analyticity in $\operatorname{Re}\left(\xi^{0}\right)>0$.

We have shown in [13] that the Schwinger 2-point function $S\left(x_{1}, x_{2}\right)$ given by (41) is reflection positive if and only if $a \mapsto G_{a a}$ is a Stieltjes function ([25]),

$$
G_{a a}=\int_{0}^{\infty} \frac{d(\rho(t))}{a+t}
$$

for a positive and non-decreasing measure $\rho$. The proof follows from the Källén-Lehmann representation of the two-point function.

The numerical results [15] exclude reflection positivity for any $\lambda>0$ (due to renormalisation). Interestingly, it thus favours the wrong-sign $\lambda \phi^{4}$-model studied in [22]. A rigorous proof that $G_{a a}$ satisfies the Stieltjes property for $\lambda<0$ is still missing, although the numerical results of [15] provide strong evidence that this will be true.

\section{Summary}

By applying the Wilson-Polchinski ideas to the noncommutative $\Phi^{4}$-theory with harmonic oscillator term we were able to solve the renormalisation problem. We obtained renormalisability to all orders of pertubation theory and reconfirmed the UV/IR-duality. Next, we showed that the running coupling constant has bounded flow to one-loop order. This led to a close collaboration with Vincent Rivasseau and his group. They proved the essential result, that at a special self-duality point, the $\beta$-function vanishes to all orders in perturbation theory.

Ward identities are the reason behind this result. They allow to decouple the hierarchy of Schwinger-Dyson equations, which allows to solve the model.

We have shown that the $\phi_{4}^{4}$-model on noncommutative Moyal space, considered in the limit $\theta \rightarrow \infty$ of extreme noncommutativity, is an exactly solvable and non-trivial matrix model. Euclidean symmetry is violated in the beginning, but we identified a limit which projects to diagonal matrices where Euclidean symmetry is restored. Surprisingly, the first consistency checks for OS positivity are passed for the only interesting interval $\left[\lambda_{c}, 0\right]$ of the coupling constant! This model is somewhat strange as 'particles' keep their momenta in interaction processes. Nevertheless, the theory is not completely trivial. We find scattering remnants from the noncommutative geometrical (i.e., matricial) substructure. Only the external matrix indices are put 'on-shell', internally all degrees of freedom contribute. We have seen that clustering is maximally violated. The interaction is insensitive to positions in different boundary components. In particular, 'particles' are never asymptotically free.

Acknowledgements Through all the years, we profited a lot from the generous support of Prof. Zeidler and the Max-Planck Institute in Leipzig, Inselstrasse. We lost an eminent Mathematician and we lost a dear elder friend. We thank Prof. Phu for his patience till we finished our review.

Funding information Open access funding provided by University of Vienna.

Open Access This article is distributed under the terms of the Creative Commons Attribution 4.0 International License (http://creativecommons.org/licenses/by/4.0/), which permits unrestricted use, distribution, and reproduction in any medium, provided you give appropriate credit to the original author(s) and the source, provide a link to the Creative Commons license, and indicate if changes were made. 


\section{References}

1. Bahns, D., Doplicher, S., Fredenhagen, K., Piacitelli, G.: On the unitarity problem in space/time noncommutative theories. Phys. Lett. B 533, 178-181 (2002)

2. Bahns, D.: The ultraviolet infrared mixing problem on the noncommutative Moyal space. arXiv:1012.3707 (2010)

3. Carleman, T.: Sur la résolution de certaines équations intégrales. Arkiv Mat. Astron. och Fysik 16, 19 (1922)

4. Disertori, M., Rivasseau, V.: Two and three loops beta function of non-commutative $\phi_{4}^{4}$ theory. Eur. Phys. J. C 50, 661-671 (2007)

5. Disertori, M., Gurau, R., Magnen, J., Rivasseau, V.: Vanishing of beta function of non commutative $\phi_{4}^{4}$ theory to all orders. Phys. Lett. B 649, 95-102 (2007)

6. Gayral, V., Gracia-bondía, J.M., Iochum, B., Schücker, T., Várilly, J.C.: Moyal planes are spectral triples. Commun. Math. Phys. 246, 569-623 (2004)

7. Gracia-Bondía, J.M., Várilly, J.C.: Algebras of distributions suitable for phase-space quantum mechanics. I. J. Math. Phys. 29, 869-879 (1988)

8. Grosse, H., Wulkenhaar, R.: The $\beta$-function in duality-covariant non-commutative $\phi^{4}$-theory. Eur. Phys. J. C 35, 277-282 (2004)

9. Grosse, H., Wulkenhaar, R.: Power-counting theorem for non-local matrix models and renormalisation. Commun. Math. Phys. 254, 91-127 (2005)

10. Grosse, H., Wulkenhaar, R.: Renormalisation of $\phi^{4}$-theory on noncommutative $\mathbb{R}^{4}$ in the matrix base. Commun. Math. Phys. 256, 305-374 (2005)

11. Grosse, H., Wulkenhaar, R.: Renormalization of $\phi^{4}$-theory on non-commutative $\mathbb{R}^{4}$ to all orders. Lett. Math. Phys. 71, 13-26 (2005)

12. Grosse, H., Wulkenhaar, R.: Progress in solving a noncommutative quantum field theory in four dimensions. arXiv:0909.1389 (2009)

13. Grosse, H., Wulkenhaar, R.: Solvable limits of a 4D noncommutative QFT. arXiv:1306.2816 (2013)

14. Grosse, H., Wulkenhaar, R.: Self-dual noncommutative $\phi^{4}$-theory in four dimensions is a nonperturbatively solvable and non-trivial quantum field theory. Commun. Math. Phys. 329, 1069-1130 (2014)

15. Grosse, H., Wulkenhaar, R.: Solvable 4D noncommutative QFT: phase transitions and quest for reflection positivity. arXiv:1406.7755 (2014)

16. Grosse, H., Wulkenhaar, R.: On the fixed point equation of a solvable 4D QFT model. Vietnam J. Math. 44, 153-180 (2016)

17. Langmann, E., Szabo, R.J.: Duality in scalar field theory on noncommutative phase spaces. Phys. Lett. B 533, 168-177 (2002)

18. Langmann, E., Szabo, R.J., Zarembo, K.: Exact solution of quantum field theory on noncommutative phase spaces. J. High Energy Phys. 0401, 17 (2004)

19. Minwalla, S., Van Raamsdonk, M., Seiberg, N.: Noncommutative perturbative dynamics. J. High Energy Phys. 0002, 20 (2000)

20. Osterwalder, K., Schrader, R.: Axioms for Euclidean Green's functions. Commun. Math. Phys. 31, 83112 (1973)

21. Osterwalder, K., Schrader, R.: Axioms for Euclidean Green's functions II. Commun. Math. Phys. 42, 281-305 (1975)

22. Rivasseau, V.: Construction and Borel summability of planar four-dimensional Euclidean field theory. Commun. Math. Phys. 95, 445-486 (1984)

23. Rivasseau, V.: From Perturbative to Constructive Renormalization. Princeton University Press, Princeton (1991)

24. Tricomi, F.G.: Integral Equations. Interscience, New York (1957)

25. Widder, D.V.: The Stieltjes transform. Trans. Am. Math. Soc. 43, 7-60 (1938) 